\title{
2 Digital Health Standort Deutschland - Brauchen wir eine Moonshot-Strategie?
}

\author{
Birgit Fischer, Kirsten Hoyer und Stefan Höcherl
}

Die digitale Transformation in Wirtschaft und Gesellschaft ist disruptiv, wenig vorhersagbar und in ihren Konsequenzen fundamental. Auch im Gesundheitswesen wandeln sich durch den Einsatz digitaler Technologien Rollenbilder und das Selbstverständnis der Akteure im Gesundheitswesen oder werden infrage gestellt. Herausfordernd dabei ist, dass digitale Services die Interaktion von Patienten, Ärzten und anderen Akteuren im Gesundheitswesen verändern. Offen ist auch noch die Frage nach der Akzeptanz der Beteiligten, der Zugänglichkeit des Gesundheitswesens für Innovationen und der gezielten Integration neuer digitaler Anwendungen, beispielsweise von Chatbots im Versorgungsmanagement oder von digitalen Expertensystemen als Unterstützungsinstrumente für Ärzte. Brauchen wir also eine MoonshotStrategie für den Digital Health Standort Deutschland?

\section{Wie tiefgreifend ist der Wandel durch die Digitalisierung und was verändert sich für Patienten?}

Digitalisierung ist kein Zukunftstrend. Sie ist Gegenwart und richtet den Wettbewerb auch im Gesundheitswesen neu aus. Die Übergänge zwischen den Versorgungsbereichen werden durchlässiger und neue Akteure mit digitalen Angeboten ergänzen traditionelle Gesundheits- und Versorgungsanbieter. Das ist eine globale Entwicklung und in manchen Gesundheitssystemen bereits weiter fortgeschritten als in Deutschland (z.B. Dänemark, Niederlande). Auch die forschenden Pharma-Unternehmen sind mit der Herausforderung konfrontiert, ihre „Kernleistung“ - die Erforschung und Entwicklung von Arzneimitteln - weiter zu entwickeln und an den neuen tech- 
nologischen Möglichkeiten und sich wandelnden Bedürfnissen der Patienten auszurichten.

\section{Zentrale Trends „Patient Empowerment“ und „Konvergenz“}

Zunehmend ist ein Zusammenwirken der Zielperspektiven von „Gesundheit erhalten“ und „Krankheit vermeiden“ sowie zwischen „traditionellen“ und „neuen“ Akteuren im Gesundheitswesen zu beobachten. Die Patientenversorgung verändert sich bereits durch neue digitale Technologien und neue individuelle Bedürfnisse. So rückt der „Point of Care“ durch mHealth und Telemedizin näher zum Patienten. Medizinische Apps oder die telemedizinische Sprechstunde stärken die Patientenmobilität und das Patienten-Engagement. Flexible, dezentrale Versorgungsformen, die den Patientenalltag besser integrieren, ergänzen bestehende Strukturen. Zeitgleich werden immer mehr digitale Services von nicht traditionellen Akteuren angeboten. „Disruptoren “ aus anderen Branchen wie der IT- und Kommunikationstechnik, der Elektroindustrie oder der Logistik entwickeln neue Gesundheitsangebote. Diese Entwicklung wird gestützt durch den Trend vom „Consumer“ hin zum anspruchsvollen, selbstbestimmten „Prosumer“. Im Gesundheitswesen wird sich in Zukunft so die Entwicklung vom „quantified self“ hin zum „qualified self“ verstärken.

\section{Forschende Pharma-Unternehmen nutzen Digitalisierung zur Optimierung bestehender Prozesse und Produkte und für neue Ansätze in Forschung und Versorgung}

\section{Digitale Vernetzung optimiert Sicherheit von Arzneimitteln}

In der Produktion von Arzneimitteln sichert und optimiert die digitale Überwachung und intelligente Vernetzung von Produktionseinheiten die Ergebnisqualität. Ein weiterer Schritt zur Verbesserung der Sicherheit von Arzneimitteln in der Versorgung sind digitale Produkt- und Gebrauchsinformationen. Beispiele hierfür sind die aktuellen Pilotprojekte unter vfa-Federführung „Gebrauchsinformation 4.0 “ und „Securpharm“. Diese verfolgen das Ziel, die Sicherheit in der Anwendung von verschreibungspflichtigen Arzneimitteln durch adressatengerechte elektronische Gebrauchsund Fachinformationen weiter zu erhöhen und den Patienten durch die lückenlose Rückverfolgbarkeit einzelner Packungen durch ein End-to-End Verifikationssystem vor illegalen Arzneimittelfälschungen in der legalen Lieferkette zu schützen.

\section{Big Data und „OMNICS-Technologien“ machen klinische Forschung effizienter}

Die „Omics-Technologien“ sind ein relativ neues biomedizinisches Forschungsgebiet, das sich mit Genomik, Proteomik und Metabolomik befasst. Eine zentrale Rolle spielen dabei große Datenmengen, für deren Verarbeitung und Auswertung Informationstechnologie (IT) eingesetzt und Big-Data-Technologien angewendet werden. Die erfolgreiche vollständige Aufschlüsselung der Genome (Genomsequenzierung) im Jahr 2003 hat die Bedeutung der Genomik in der Medizin bereits deutlich gestärkt. Der nächste Schritt geht nun hin zur Proteomik, um die individuelle Proteinkonstellation und die damit verbundenen Stoffwechselprozesse besser zu verstehen. Die 
Genomsequenzierung und der Einsatz von diagnostischen Tests durch Big-Data-Anwendungen ermöglichen deutliche Fortschritte in der Forschung. So können dadurch z.B. klinische Studien verbessert und neue Therapieansätze gefunden werden.

\section{„Personalisierte Medizin“ steigert Qualität in der Versorgung}

Die Kombination von moderner Diagnostik und innovativem Arzneimittel ermöglicht eine „Personalisierte Medizin“, die die Wirkungsprofile der Arzneimittel weiter schärft und dem Patienten eine bestmöglich an seine Erkrankung angepasste Therapie bietet. Der behandelnde Arzt wird dadurch bei der Festlegung der für den jeweiligen Patienten am besten geeigneten Therapie und der Überprüfung des Therapieerfolgs weiter unterstützt. Um geeignete Biomarker zu identifizieren und zu validieren, ist ein besonderer Forschungsaufwand nötig. Biobanken schaffen dabei die Verknüpfung von Patientendaten aus klinischen Studien mit genetischen Daten (Sequenzierungsdaten).

\section{Digitale Angebote und Datennutzung steigern Therapietreue und schaffen neue Evidenz}

Das Sammeln (Tracking und Register) und Auswerten von (Echtzeit-)Daten aus dem Versorgungsalltag können zusätzliche Nachweise (Evidenz) zum Nutzen von Arzneimitteln schaffen. Ergänzende digitale Angebote zum Beispiel Apps und Plattformen, die in Zusammenarbeit mit Krankenkassen und Ärzten angeboten werden, bieten Patienten persönliche Schulungsprogramme zu ihrer Erkrankung oder zu alltagsrelevanten Therapiehinweisen (Disease Awareness und Patient Support Programme). Das unterstützt Patienten aktiv, mit Unsicherheiten im Zusammenhang mit ihrer Erkrankung und der Therapie umzugehen und ihre Therapietreue zu erhöhen. Zudem werden die Sicherheit und Qualität der Arzneimitteltherapie durch ein medizinisches Monitoring der Behandlungsdaten, das immer häufiger in Echtzeit erfolgt, weiter verbessert. Zusammenfassend dazu siehe auch Abbildung 1 zu Chancen und Anwendungen von der Forschung bis zur Patientenversorgung.

\section{Kooperationen führen zu digitalen Versorgungsinnovationen}

Ein Beispiel ist die intelligente Verknüpfung von Arzneimittel und Medizinprodukt zur Verbesserung der Versorgung von chronischen Patienten. Sogenannte Smart Devices können medizinische Daten, z.B. Blutzuckerwerte, digital messen und aufzeichnen. Ein Smart Service entsteht dann durch das sichere sektorenübergreifende Teilen der Daten mit dem zuständigen medizinischen Fachpersonal zur Beobachtung des Therapieerfolgs in Echtzeit über entsprechende IT-Plattformen. Dadurch wird eine individuellere Anpassung an die Patientenbedürfnisse und eine Verbesserung der Medikationssteuerung möglich. Neue Netzwerke entstehen z.B. auch durch die Ergänzung der Versorgung durch Start-ups. Forschende Pharma-Unternehmen unterstützen solche Ansätze durch unterschiedliche Kooperationsformate wie Acceleratoren oder Digital Labs. So entstehen und verfestigen sich Netzwerke und InnovationsCluster wie z.B. in Berlin für den Gesundheitsbereich. 


\section{Von der Vision zur Agenda! Ausbau des Digital Health Standorts Deutschland und Verbesserung der Patientenversorgung}

Im internationalen Wettbewerb ist die deutsche Gesundheitswirtschaft auf einen starken „Digitalisierungs-Standort“ angewiesen. Ein Erfolgsfaktor sind Rahmenbedingungen, die die akuten Fragen, z.B. die Gestaltung von Qualitätsstandards für eHealth-Angebote, die Integration neuer digitaler Angebote in die gesetzliche Regelversorgung und die Einbindung und Beteiligung von Patienten, aufgreifen.

\section{Patientennutzen bei digitalen Innovationen in den Mittelpunkt stellen}

Zentraler Prüfstein für die Digitalisierung im Gesundheitswesen muss der Patientennutzen sein. Digitale Angebote können die Patientenautonomie, den individuellen Nutzen für den Patienten und die Therapietreue (Adhärenz) steigern, z.B. wenn chronische Patienten weiterführende digitale Informationsangebote zur Unterstützung ihrer Therapie erhalten. Essenziell für die praktische Umsetzung des informationellen Selbstbestimmungsrechts des Patienten und die Steigerung der Effizienz an den Schnittstellen im System ist eine effektive elektronische Patientenakte, das zeigen Beispiele anderer europäischer Gesundheitssysteme. Die mit dem „eHealth-Gesetz“ geplante regelhafte Einführung in Deutschland bis 2019 ist daher ein unerlässlicher Schritt zur Stärkung der Patientenzentrierung.

\section{Chancen der intelligenten Vernetzung von Gesundheits- und Forschungsdaten aktiv nutzen}

Die intelligente Verarbeitung von Forschungs- und Gesundheitsdaten bietet enorme Chancen für die Verbesserung der Patientenversorgung und ein nachhaltigeres Cesundheitswesen. Das Ziel sollte eine Harmonisierung der diversen Regelungen für eine sichere Vernetzung und die vertrauenswürden Nutzung von Daten sein. Dies würde z.B. die klinische Forschung und die translationale Medizin entscheidend stärken. Vor diesem Hintergrund ist die EU-Datenschutzgrundverordnung (EUDSGVO) vom 24.05.2016 ein Meilenstein des europäischen Datenschutzrechts und spielt bei der politischen Rahmensetzung für die digitale Transformation diverser Branchen und für den Umgang mit dem „Rohstoff Daten“ eine zentrale Rolle.

\section{Aktionsräume für Zusammenarbeit schaffen, um Vertrauen zu fördern}

Eine Herausforderung im Vergleich zu anderen Gesundheitssystemen ist für das deutsche System nach wie vor eine gewisse „Anti-Vernetzungskultur“. Um die mit Echtzeit-Analysen und mit Big-Data-Technologien verbundenen Chancen schrittweise zu realisieren, ist aber eine stärkere Verständigung der beteiligten Akteure notwendig. Die Veränderungen in der Versorgungslandschaft durch digitale Angebote und medizinisch-technologischen Fortschritt führen verständlicherweise oft zu Verunsicherung und häufig zu einer risikozentrierten, technischen Fachdiskussion. Für mehr Vertrauen in die Digitalisierung im Gesundheitswesen müssen die Chancen stärker diskutiert und neue Versorgungsansätze in Aktionsräumen erprobt und der Nutzen praktisch erlebt werden. 